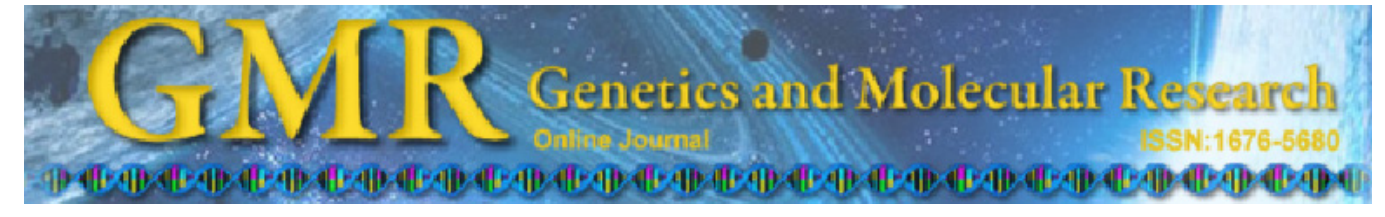

\title{
Isolation and characterization of microsatellite DNA loci from the peanut worm, Sipunculus nudus
}

\author{
Q.H. Wang, Y.S. Guo, Z.D. Wang, X.D. Du and Y.W. Deng \\ Fisheries College, Guangdong Ocean University, Zhanjiang, China
}

Corresponding author: X.D. Du

E-mail: zjdxd@21cn.com

Genet. Mol. Res. 11 (2): 1662-1665 (2012)

Received August 9, 2011

Accepted December 9, 2011

Published June 15, 2012

DOI http://dx.doi.org/10.4238/2012.June.15.15

\begin{abstract}
Sipunculus nudus, the peanut worm, is the best known species in its genus. This unsegmented subtidal marine worm is consumed in some parts of Asia and is also used as fish bait. We found 20 microsatellite DNA markers for $S$. nudus. The number of alleles per polymorphic locus ranged from two to seven in a sample of 39 individuals. Observed and expected heterozygosities per polymorphic locus varied from 0.103 to 1.000 and from 0.307 to 0.771 , respectively. Five loci showed significant departure from Hardy-Weinberg equilibrium after sequential Bonferroni's correction. No significant linkage disequilibrium between pairs of loci was found. These microsatellite markers will provide useful tools for investigating genetic population structure, population history and conservation management of $S$. nudus.
\end{abstract}

Key words: Sipunculus nudus; FIASCO microsatellites; Peanut worm; Genetic diversity 


\section{INTRODUCTION}

Sipunculus nudus (peanut worm) is considered a cosmopolitan species found in all oceans from intertidal zones to a depth of $900 \mathrm{~m}$. It is the most known species in the genus Sipunculus. As it is common and easily collected along the Atlantic and Mediterranean coasts of Europe, it has been studied for many decades by developmental biologists, physiologists, and biochemists (Cutler, 1994). The body of the adult worm is around $15 \mathrm{~cm}$ in length but can reach up to $25 \mathrm{~cm}$ in some cases. $S$. nudus are used by humans in two different ways, as food or as fish bait. In parts of southern China such as the Provinces of Guandong, Guangxi, Hainan, and Fujian, S. nudus is collected, cleaned of its innards, and eaten as a local delicacy. Besides for humans, sipunculans are a source of food for many fishes. Among invertebrates, there are a few such as gastropods and asteroids that may feed on sipunculans as part of their diet (Kohn, 1975). So far, studies have focused on the biology (Wang et al., 2008), histology (Cao et al., 2009), artificial propagation (Li et al., 2004), and phylogeny (Schulze et al., 2007; Mwinyi, 2009) of sipunculan worms. In the present study, we developed 20 microsatellite loci isolated from a dinucleotide-enriched genomic library of $S$. nudus using the fast isolation by AFLP of sequences containing repeats (FIASCO) protocol of Zane et al. (2002).

\section{MATERIAL AND METHODS}

A sample of 39 individuals of $S$. nudus were randomly collected from intertidal zones of Liusha Town, Zhanjiang County, Guangdong Province, China. Genomic DNA was extracted from the muscle tissue using a standard phenol-chloroform procedure (Sambrook et al., 1989). Total genomic DNA (about $500 \mathrm{ng}$ ) obtained was completely digested with $\mathrm{MseI}$ and then ligated to an MseI AFLP adaptor (MA-1: 5'-GAC GAT GAG TCC TGA G-3'; MA-2: 5'-TAC TCA GGA CTC AT-3'). A diluted digestion-ligation mixture (1:10) was amplified with adaptor-specific primers (MseP: 5'-GAT GAG TCC TGA GTA A-3'). Amplified DNA fragments, with a size range of 200-1000 bp, were enriched for repeats by magnetic bead selection with a 5'-biotinylated $(\mathrm{AC})_{15}$ probe. Enriched fragments were again amplified with adaptorspecific primers. Polymerase chain reaction (PCR) products were purified using an EZNA Gel Extraction kit (Omega Bio-Tek). Purified DNA fragments were ligated into the pMD-18T vector $(\mathrm{TaKaRa})$, and transformed into DH5 $\alpha$ cells. Positive clones were tested by PCR using $(\mathrm{AC})_{15}$ and MseP as primers. In total, 99 clones with positive inserts were sequenced with an ABI PRISM 3730xl DNA sequencer. A total of 67 sequences were found to contain microsatellite repeats, and 41 of them were selected for designing locus-specific primers, using the Oligo 6 software package (Molecular Biology Insights, Inc.). Polymorphisms of all 16 microsatellite loci were assessed in 39 samples of $S$. nudus from Liusha Town. These microsatellite sequences have been deposited in GenBank (HM241349-HM241416).

The PCRs were performed in a $10-\mu \mathrm{L}$ reaction mixture containing 30-50 ng genomic DNA, $0.5 \mu \mathrm{M}$ of each primer, $1 \mu \mathrm{L} 10 \mathrm{X}$ PCR buffer (10 mM Tris- $\mathrm{HCl}, \mathrm{pH} 8.3), 50 \mathrm{mM} \mathrm{KCl}$, $\left.1.5 \mathrm{mM} \mathrm{MgCl}_{2}\right), 0.2 \mathrm{mM}$ of each dNTP and $1 \mathrm{U}$ Takara Taq polymerase. PCR runs began with an initial denaturation step at $94^{\circ} \mathrm{C}$ for 3 min followed by 30 cycles at $94^{\circ} \mathrm{C}$ for $30 \mathrm{~s}, 30 \mathrm{~s}$ at the annealing temperature (Ta), and $72^{\circ} \mathrm{C}$ for $45 \mathrm{~s}$, and a final extension step at $72^{\circ} \mathrm{C}$ for 5 min. PCR products were separated on $8 \%(\mathrm{w} / \mathrm{v})$ polyacrylamide gels using the $\mathrm{pBR} 322 / B s u \mathrm{R}$ marker (MBI Fermentas) by silver staining. Heterozygosis values, tests of Hardy-Weinberg 
and genotypic equilibrium were calculated with the package GENEPOP3.4 (10,000 dememorizations, 100 batches, 5000 iterations per batch) (Raymond and Rousset, 1995).

\section{RESULTS AND DISCUSSION}

Four loci (Snu08, Snu10, Snu12, and Snu14) were monomorphic. Descriptive statistics for the microsatellites are given in Table 1. In polymorphic loci, the numbers of alleles ranged from 2 to 7 and observed heterozygosity from 0.103 to 1.000 . Five microsatellite loci deviated significantly from Hardy-Weinberg expectations including a Bonferroni's correction (Bonferroni, 1935), indicating either that null alleles were present or that the sample size was small. No significant disequilibrium within samples was found, indicating that genotypes at microsatellite pairs that appeared randomly associated and microsatellites were inherited in a Mendelian fashion.

\begin{tabular}{|c|c|c|c|c|c|c|c|c|c|c|}
\hline Clone No. & Locus & Primer sequence $\left(5^{\prime}-3^{\prime}\right)$ & $\mathrm{Ta}\left({ }^{\circ} \mathrm{C}\right)$ & Size (bp) & Repeat motif & $N_{\mathrm{A}}$ & $N_{\mathrm{E}}$ & $H_{\mathrm{O}}$ & $H_{\mathrm{E}}$ & HWE P \\
\hline 388 & Snu01 & $\begin{array}{l}\text { ACCACCAAGTAAGAGGCA } \\
\text { TATTCCCAGGTTCATTCG }\end{array}$ & 53 & $272 \sim 298$ & $(\mathrm{gt})_{7}$ & 3.0 & 1.9 & 0.641 & 0.483 & 0.034 \\
\hline 354 & Snu02 & $\begin{array}{l}\text { TCGGCGTACCAATGTAGA } \\
\text { GATTCCGTTCACGACTGC }\end{array}$ & 51 & $130 \sim 176$ & $(\mathrm{gt})_{5} \mathrm{~N}(\mathrm{tg})_{7}$ & 4.0 & 2.3 & 0.757 & 0.564 & 0.010 \\
\hline 271 & Snu03 & $\begin{array}{l}\text { CGGGTAGGAACACCAAAG } \\
\text { CGAAATGCCCTGAAAATC }\end{array}$ & 51 & $150 \sim 176$ & $(g t)_{8}$ & 2.0 & 2.0 & 0.897 & 0.500 & $0.000^{*}$ \\
\hline 222 & Snu04 & $\begin{array}{l}\text { ATTATTCGTATGTGGCTCC } \\
\text { AATCCGTAGAAGATGCTG }\end{array}$ & 53 & $194 \sim 210$ & $(\operatorname{tg})_{5}$ & 4.0 & 1.9 & 0.282 & 0.476 & $0.000^{*}$ \\
\hline 216 & Snu05 & $\begin{array}{l}\text { GGAGGCTATGCTTTCATT } \\
\text { TTTACTTATTCCACCCGTA }\end{array}$ & 50 & $292 \sim 324$ & $(\mathrm{gt})_{6}$ & 4.0 & 2.7 & 0.395 & 0.623 & 0.008 \\
\hline 215 & Snи06 & $\begin{array}{l}\text { GCACGAATTGACAGAAAT } \\
\text { GACTAAAACGTAACCGATG }\end{array}$ & 48 & $96 \sim 130$ & $(\operatorname{tg})_{5} \mathrm{~N}(\mathrm{tg})_{6}$ & 4.0 & 2.7 & 0.447 & 0.623 & $0.000^{*}$ \\
\hline 166 & Snи07 & $\begin{array}{l}\text { GTTCATCCCCGTTCGTGA } \\
\text { CGGTGTCCGTATTGTGGT }\end{array}$ & 53 & $288 \sim 306$ & $(\mathrm{gt})_{7}$ & 2.0 & 1.4 & 0.378 & 0.307 & 0.156 \\
\hline 158 & Snu08 & $\begin{array}{l}\text { ATCCTCACATCACCGAGTC } \\
\text { CCGAAACCTACCTTAGCG }\end{array}$ & 53 & 258 & $(\mathrm{gt})_{5}$ & 1.0 & 1.0 & 0.000 & 0.000 & - \\
\hline $135-1$ & Snи09 & $\begin{array}{l}\text { TGGAAAATACACTCAAGAAA } \\
\text { AGATAAGCTGGTAGAGGATG }\end{array}$ & 50 & $232 \sim 262$ & $(\operatorname{tg})_{5}$ & 3.0 & 1.8 & 0.436 & 0.429 & 0.245 \\
\hline 397 & Snu 10 & $\begin{array}{l}\text { ATTTATAGAAAGGTTACAGCAG } \\
\text { TATTTACGGGGTCAGGGT }\end{array}$ & 53 & 274 & $(\mathrm{gt})_{5}$ & 1.0 & 1.0 & 0.000 & 0.000 & - \\
\hline $346-2$ & Snu11 & $\begin{array}{l}\text { CGTACAGGCGCTAAATAT } \\
\text { GATGAATTGTATGCGTCAC }\end{array}$ & 48 & $172 \sim 190$ & $(\mathrm{gt})_{10}$ & 7.0 & 4.4 & 0.343 & 0.771 & $0.000^{*}$ \\
\hline $330-2$ & Snu 12 & $\begin{array}{l}\text { GCGTTCATCCCCGTTCGT } \\
\text { CAAGCCTCCGCCCTGTAA }\end{array}$ & 60 & 302 & $(\mathrm{gt})_{6}$ & 1.0 & 1.0 & 0.000 & 0.000 & - \\
\hline $330-1$ & Snu 13 & $\begin{array}{l}\text { TTGGGCTTACCACCTACA } \\
\text { ACGGGGTCAAGAACATCA }\end{array}$ & 49 & $148 \sim 192$ & $(\mathrm{at})_{5}$ & 3.0 & 1.5 & 0.359 & 0.310 & 0.601 \\
\hline 288 & Sпи 14 & $\begin{array}{l}\text { CATTATTGCCTCCACTTG } \\
\text { ACCTTTTCTGGGATTGTC }\end{array}$ & 51 & 198 & $(\operatorname{tg})_{5}$ & 1.0 & 1.0 & 0.000 & 0.000 & - \\
\hline 280 & Snu 15 & $\begin{array}{l}\text { CCGAAACTGACCTGTGAT } \\
\text { GTAGCCTAATGGCGAACT }\end{array}$ & 58 & $246 \sim 276$ & $(\operatorname{tg})_{5}$ & 3.0 & 1.1 & 0.103 & 0.098 & 0.990 \\
\hline 266 & Snu16 & $\begin{array}{l}\text { TCCGCTAGGAGGGTATGC } \\
\text { AGACGGCGTTTCAGTTTG }\end{array}$ & 56 & $262 \sim 398$ & $(\operatorname{tg})_{6}$ & 5.0 & 1.6 & 0.471 & 0.387 & 0.976 \\
\hline 236 & Snи 17 & $\begin{array}{l}\text { TGTCCGATTGCCTACTGA } \\
\text { ACACCAAATGCTAACAGAT }\end{array}$ & 45 & $168 \sim 210$ & $(\mathrm{gt})_{7}$ & 6.0 & 3.3 & 0.795 & 0.699 & 0.225 \\
\hline 186 & Sпи 18 & $\begin{array}{l}\text { TGTCTCCGCTCTTCTCAT } \\
\text { TCATAAATAAACCCCTGTAA }\end{array}$ & 49 & $122 \sim 134$ & $(\mathrm{ca})_{5}$ & 2.0 & 2.0 & 1.000 & 0.500 & $0.000^{*}$ \\
\hline 92 & Sпи 19 & $\begin{array}{l}\text { ACAAAGAAAGACGAAGGC } \\
\text { CGTCCCAACTTTTGAGTA }\end{array}$ & 55 & $174 \sim 221$ & $(\mathrm{gt})_{5}$ & 6.0 & 1.6 & 0.436 & 0.375 & 1.000 \\
\hline 25 & Sпи 20 & $\begin{array}{l}\text { GCCAGGAGGGTATGTGAT } \\
\text { TGATGGATTCTTCTTTGGA }\end{array}$ & 49 & 139 155 & $(\operatorname{tg})_{7}$ & 2.0 & 1.5 & 0.410 & 0.326 & 0.107 \\
\hline
\end{tabular}

$\mathrm{Ta}=$ annealing temperature; $N_{\mathrm{A}}=$ number of alleles; $N_{\mathrm{E}}=$ number of effective alleles; $H_{\mathrm{O}}=$ observed heterozygosity; $H_{\mathrm{E}}=$ expected heterozygosity; $\mathrm{HWE}=$ Hardy-Weinberg equilibrium. $\mathrm{P}<0.0025 ;(-)=$ monomorphic. 


\section{REFERENCES}

Bonferroni CE (1935). 11 Calcolo Delle Assicurazioni su Gruppi di Teste. In: Studi in Onore Del Professore Salvatore Ortu Carboni, Rome, 13-60.

Cao X-W, Huang R-N, Wang Q-H and Du X-D (2009). Anatomical and histological study on digestive system of Sipunculus nudus. J. Guangdong Ocean Univ. 29: 6-10.

Cutler EB (1994). The Sipuncula, their Systematics, Biology, and Evolution. Cornell University, Ithaca.

Kohn AJ (1975). Predation on Sipunculans. In: Proceedings of the International Symposium on the Biology of the Sipuncula and Echiura, Kotor (Rice ME and Todoroviae M, eds.). Nauèno Delo Press, Belgrade, 313-333.

Li JS, Feng DQ and Zhou SQ (2004). Primary studies on reproductive biology of Sipunculus nudus. J. Hangzhou Teach. Coll. (Natural Science Edition) 3: 136-139.

Mwinyi A, Meyer A, Bleidorn C, Lieb B, et al. (2009). Mitochondrial genome sequence and gene order of Sipunculus nudus give additional support for an inclusion of Sipuncula into Annelida. BMC Genomics 10: 27.

Raymond M and Rousset F (1995). GENEPOP (version 1.2): population genetics software for exact tests and ecumenism. J. Hered. 86: 248-249.

Sambrook J, Fritsch EF and Maniatis T (1989). Molecular Cloning: A Laboratory Manual. 2nd edn. Cold Spring Harbor Laboratory Press, New York.

Schulze A, Cutler EB and Giribet G (2007). Phylogeny of sipunculan worms: A combined analysis of four gene regions and morphology. Mol. Phylogenet. Evol. 42: 171-192.

Wang Q-H, Du Y-H and Lin S-L (2008). Karyotype analysis of Sipunculus nudus. Chin. J. Zool. 43: 95-98.

Zane L, Bargelloni L and Patarnello T (2002). Strategies for microsatellite isolation: a review. Mol. Ecol. 11: 1-16. 